\title{
A HEURÍSTICA DA DISPONIBILIDADE E A IDENTIFICAÇÃO DO CICLO ECONÓMICO: UMA ANÁLISE BASEADA NOS DADOS DE CONFIANÇA DOS CONSUMIDORES EM PORTUGAL
}

\section{THE HEURISTIC OF AVAILABILITY AND IDENTIFICATION \\ OF THE ECONOMIC CYCLE: AN ANALYSIS BASED ON CONSUMER CONFIDENCE DATA IN PORTUGAL}

\section{Luís Bernardes ${ }^{1}$}

\begin{abstract}
Resumo: A Heurística da Disponibilidade, originalmente designada por Daniel Kahneman e Amos Tversky, descreve o fenómeno comportamental segundo o qual os indivíduos antecipam a frequência de um evento com base na facilidade com que se conseguem lembrar de exemplos desse evento. Este estudo analisa de que forma este "efeito disponibilidade" pode ajudar a identificar e a antecipar o ciclo económico, utilizando como medida desse efeito a diferença das respostas dos consumidores portugueses às questões sobre a "evolução da situação financeira do agregado familiar" e a "evolução da situação económica do país" constantes dos inquéritos mensais de confiança. $O$ estudo conclui que o "efeito disponibilidade" aumenta a robustez da correlação contemporânea entre os indicadores de confiança e o crescimento económico, em parte porque mitiga as limitações decorrentes do chamado "efeito referência", segundo o qual o nível de confiança dos indivíduos é influenciado, a cada momento, por um "ponto de referência", nem sempre constante. $O$ estudo conclui, ainda, que o "efeito disponibilidade" apresenta uma elevada correlação desfasada com a atividade económica, podendo contribuir para reforçar o papel dos índices de confiança como indicadores avançados do ciclo económico. Um argumento possível para este resultado é que o "efeito disponibilidade" capta simultaneamente o volume de informação existente sobre o ciclo económico e a previsível resposta dos indivíduos a essa informação.
\end{abstract}

Palavras-chave: Heurísticas, Disponibilidade, Ciclo Económico, Indicadores Avançados.

1 Prof Auxiliar - Departamento de Economia, Gestão e Ciências Sociais da Universidade Católica Portuguesa. Email: luis.g.bernardes@gmail.com 


\begin{abstract}
The Availability Heuristic, originally labeled by Daniel Kahneman and Amos Tversky, describes the behavioral phenomenon according to which individuals anticipate the frequency of an event based on how easily one can remember examples of that event. This study analyzes how this "availability effect" can help to identify and anticipate the economic cycle, using as a measure of this effect the difference in the responses of Portuguese consumers to the questions about the "evolution of the financial situation of the household" and the "evolution of the country's economic situation" in the monthly confidence surveys. The study concludes that the "availability effect" enhances the robustness of the contemporary correlation between confidence indicators and economic growth, in part because it mitigates the limitations arising from the so-called "reference effect" according to which the level of confidence of individuals is influenced, at any moment, by a "reference point", not always constant. The study also concludes that the "availability effect" presents a high lagged correlation with economic activity, and may contribute to reinforce the role of confidence indexes as leading indicators of the economic cycle. One possible argument for this result is that the "availability effect" simultaneously captures the volume of information about the economic cycle and the individuals' predictable response to that information.
\end{abstract}

Keywords: Heuristics, Availability, Economic Cycle, Leading Indicators.

\title{
INTRODUÇÃO
}

Os resultados dos inquéritos de confiança aos consumidores são, frequentemente, utilizados para identificar e antecipar o ciclo económico, sendo tipicamente uma variável importante na construção de indicadores coincidentes e avançados da atividade económica: veja-se, por exemplo, com relevância direta para o caso português, o indicador avançado da OCDE (OCDE, 2018) ou o indicador coincidente do Banco de Portugal (Rua, 2004).

A conveniência temporal justifica, em parte, esta utilização, dado que os índices de confiança são dos primeiros indicadores, relacionados com a atividade económica, a serem divulgados, antecedendo em muito os valores do produto interno bruto (PIB) e de outros indicadores relevantes, como a produção industrial, os índices de atividade no retalho, serviços e construção ou a taxa de desemprego. No caso português, só a divulgação das vendas mensais de veículos automóveis rivaliza, em celeridade, com os índices de confiança, como indicador da atividade económica. Talvez mais relevante, contudo, é o argumento de que a confiança dos agentes 
económicos tende a refletir e a influenciar o seu próprio comportamento e, consequentemente, o desempenho corrente e futuro da economia, dando aos dados da confiança um caráter de indicador coincidente e avançado do crescimento económico (isto é, tendem a conter sinais relevantes sobre o ciclo e sobre a sua evolução próxima).

Apesar de tudo, os estudos empíricos relativos a esta matéria não são conclusivos quanto à relevância dos índices de confiança dos consumidores como indicadores do ciclo económico, em especial como indicadores avançados do ciclo (algumas referências habituais a este respeito são Bondt e Schiaffi, 2015; Carroll et al., 1994; Golinelli e Parigi, 2004; Ludvigson, 2004 e Matsusaka e Sbordone, 1995).

Um dos problemas identificados nestes estudos empíricos tem a ver com fenómenos comportamentais, que influenciam simultaneamente a qualidade dos inquéritos e o significado efetivo das respostas dos inquiridos (ver, desde logo, Acemoglu e Scott, 1994).

O nosso artigo baseia-se, precisamente, na influência que os fenómenos comportamentais podem ter nas respostas aos inquéritos de confiança, para sugerir uma melhoria na forma de utilizar os resultados desses inquéritos como indicadores do ciclo económico. Neste estudo, concentramo-nos, essencialmente, em dois fenómenos comportamentais, originalmente propostos por Daniel Kahneman e Amos Tversky e posteriormente consubstanciados e desenvolvidos por uma vasta literatura: "efeito referência" (reference-dependent preferences) e "efeito disponibilidade" (availability effect). Na bibliografia que acompanha este artigo fazemos menção resumida a alguma desta literatura, com base na sua natureza seminal ou de referência.

A primeira das limitações comportamentais que invocamos neste estudo decorre da noção de que o sentimento dos indivíduos reflete, em larga medida, uma comparação com a sua experiência recente (o chamado "efeito referência"). Isto significa que a relação entre a taxa de crescimento do PIB e os valores absolutos dos índices de confiança dificilmente será uniforme ao longo do tempo. Por exemplo, a manutenção prolongada de um dado ritmo de crescimento pode ser, a partir de certa altura, encarada de forma neutral ou pouco entusiasta pelos agentes, ainda que a taxa de crescimento do PIB seja claramente positiva; pelo contrário, uma súbita melhoria da atividade económica pode ser percebida pelos indivíduos como algo de muito favorável, ainda que o ritmo de crescimento do PIB seja, em termos absolutos, baixo ou até negativo. 
A segunda limitação tem a ver com o facto de que o nível de confiança dos indivíduos é determinado não apenas pela sua vivência pessoal, mas também por fenómenos externos, cuja correlação com o ciclo económico varia de caso para caso: fatores como a instabilidade política ou social e a cobertura mediática podem influenciar seriamente a confiança das pessoas ou a sua perceção sobre o ciclo económico e, por isso, o seu comportamento. Ao impacto que esta influência externa tem na formação de opinião dos indivíduos podemos chamar "efeito disponibilidade", tomando como referência a chamada heurística da disponibilidade, que traduz a noção de que os indivíduos tendem a julgar a frequência ou a probabilidade de um evento pela facilidade com que conseguem recordar exemplos desse evento ou pelo volume e tipo de informação recentemente adquirida sobre o mesmo.

Este artigo visa dar um contributo para a compreensão da relação entre os dados da confiança e o ciclo económico, fazendo uso prioritário do "efeito disponibilidade". O argumento base é simples: quanto maior o "efeito disponibilidade", mais elevada é a probabilidade de que o mesmo contenha informação factual sobre o ciclo ou que afete o comportamento dos agentes de forma a influenciar significativamente a evolução do ciclo económico.

A métrica que utilizamos para quantificar o "efeito disponibilidade" é a diferença entre os valores das respostas dadas a duas das questões que integram os inquéritos mensais de confiança aos consumidores: "Em sua opinião, a situação financeira do seu lar (agregado familiar), nos próximos 12 meses irá (...)" (variável SFAM) e "Em sua opinião, a situação económica geral do País, nos próximos 12 meses irá (...)" (variável SPAÍS) ${ }^{2}$.

O comportamento da variável SFAM está muito dependente da situação pessoal. Já a variável SPAÍS tende a incorporar, adicionalmente, informação genérica sobre a situação económica proveniente de fontes externas, como os relatos de terceiros, a comunicação social ou o debate político. Esta variável ajuda a identificar os sinais prevalentes do ciclo num dado momento, mas tem também características de indicador avançado, uma vez que a informação geral sobre a economia pode

\footnotetext{
2 A diferença entre as respostas dadas pelos inquiridos a questões sobre finanças pessoais e a questões sobre a situação geral da economia é, aliás, um dos aspetos mais valorizados por Dominitz e Manski (2004) no estudo efetuado sobre como elaborar índices de confiança.
} 
influenciar o comportamento dos indivíduos, com impacto relevante na atividade económica.

Uma vantagem de utilizar a diferença entre SPAÍS e SFAM (em vez de SPAÍS ou SFAM isoladamente) é que, desta forma, consegue-se mitigar o "efeito referência", como argumentamos mais à frente. Além disso, é de esperar que quanto maior for a diferença entre SPAÍS e SFAM, maior a probabilidade de que o ciclo económico esteja a ser adequadamente captado ou influenciado pelo "efeito disponibilidade". Estas duas vantagens sugerem que a correlação estatística entre o crescimento da economia e a diferença entre SPAÍS e SFAM pode ser significativa e relativamente estável ao longo do tempo, quer em termos coincidentes quer desfasados.

O resto do artigo está organizado da seguinte forma. No capítulo 2 desenvolvemos alguns dos conceitos mais relevantes para o nosso estudo, em particular os fenómenos comportamentais e os indicadores económicos utilizados como variáveis endógenas. No capítulo 3 explicamos brevemente a metodologia utilizada para testar as hipóteses propostas na introdução deste artigo e apresentamos os principais resultados, que no essencial validam essas hipóteses. O capítulo 4 conclui e propõe aspetos onde a investigação em causa pode ser desenvolvida.

\section{CONCEITOS E OUTRAS NOTAS PRÉVIAS}

\subsection{Heurísticas}

O conceito de heurísticas foi proposto, originalmente, por Kahneman e Tversky, para definir os processos ou regras simplificadoras que os indivíduos utilizam para fazer escolhas, tomar decisões ou avaliar cenários (Tversky e Kahneman, 1994 e Gilovich et al., 2002). Um dado importante relativo a estas regras é que as mesmas divergem frequentemente dos pressupostos utilizados pelos modelos tradicionais económicos e financeiros, o que explica a sua incorporação crescente na investigação levada a cabo nestas áreas.

Neste artigo damos particular atenção à heurística da disponibilidade, já definida anteriormente: os consumidores tendem a julgar a probabilidade de um evento pela facilidade com que conseguem recordar exemplos desse evento ou pelo volume e tipo de informação recentemente adquirida sobre o mesmo (ver Tversky e Kahneman, 1972 e, entre outros, Rabin, 1998 e Schwarz et al., 1991). 
Além disso, para reforçar alguns argumentos, recorremos também aos conceitos de "ponto de referência" e, com menor ênfase, de "aversão às perdas". O "ponto de referência" reflete a tese de que os indivíduos avaliam cenários em função de uma referência, que é dada, no essencial, pelo resultado relativamente ao qual o indivíduo é indiferente no momento dessa avaliação (ver Kahneman e Tversky, 1979; Tversky e Kahneman, 1981 e, para desenvolvimentos mais concretos, Bateman et al., 1997; Kahneman et al., 1991 e Rabin, 1998). Sempre que o cenário proposto for encarado como favorável relativamente ao "ponto de referência", esse cenário é tratado como um ganho. No entanto, se o cenário for percebido como menos vantajoso do que o "ponto de referência", então é avaliado como uma perda. Note-se que a definição e o valor do "ponto de referência" podem variar substancialmente, conforme a circunstância e ao longo do tempo: pode ser o status-quo, a experiência recente, uma determinada expetativa ou o preço que foi pago por um bem. No caso específico das respostas dos consumidores ao inquérito de confiança, o "ponto de referência" tende a ser a vivência recente dos indivíduos, com as consequências já mencionadas na introdução deste artigo. A "aversão às perdas" refere-se à tendência que os indivíduos têm para valorizar perdas face ao "ponto de referência" bastante mais do que valorizam ganhos, gerando assim uma assimetria valorativa entre variações absolutas de valor idêntico, dependendo de se tratarem de ganhos ou de perdas (ver Tversky e Kahneman, 1991 e, por exemplo, Bowman et al., 1999, Kahneman et al., 1991 e Rabin, 1998).

\subsection{O Índice de Confiança dos Consumidores}

$\mathrm{O}$ inquérito de conjuntura aos consumidores em Portugal é realizado mensalmente pelo Instituto Nacional de Estatística (INE) e é composto por 11 questões relacionadas com a perceção, expetativas ou intenções dos indivíduos relativamente a tópicos como a evolução da situação familiar e da economia em geral, a capacidade e as intenções de poupança, a evolução dos preços, o desemprego e as intenções de consumo de bens duradouros. As respostas a estas questões são recolhidas, maioritariamente, nos primeiros dias de cada mês e os resultados, devidamente tratados e corrigidos de sazonalidade, são divulgados pelo INE e pela Comissão Europeia (Directorate-General Financial and Economic Affairs, ECFIN) no final do próprio mês em que foram recolhidas as respostas.

O valor do índice de confiança dos consumidores, relativo a cada mês, é obtido através da média ponderada do saldo das respostas extremas 
(sre) dadas a quatro dessas questões: "situação financeira do agregado familiar nos próximos 12 meses", "situação económica no país nos próximos 12 meses", "desemprego no país nos próximos 12 meses" e "capacidade de poupar nos próximos 12 meses"3.

Neste artigo fazemos uso dos valores do índice de confiança dos consumidores (ICC) e de duas das suas componentes: "situação económica no país nos próximos 12 meses" (SPAÍS) e "situação financeira do agregado familiar nos próximos 12 meses" (SFAM). Contudo, como já explicado, acrescentamos à análise a diferença entre o valor das respostas sobre a "situação económica no país" e sobre a "situação financeira do agregado familiar", sendo que o valor absoluto dessa diferença (DIF_SPSF) é utilizado como medida do "efeito disponibilidade" no que respeita à perceção dos consumidores sobre o ciclo económico.

Como já referido, para além de ilustrar e ajudar a quantificar o "efeito disponibilidade", a utilização da diferença entre as variáveis SPAÍ́S e SFAM tem ainda a vantagem de mitigar o "efeito referência". Quando calculado de forma isolada, o valor das variáveis SPAÍS e SFAM é influenciado pelo "ponto de referência" dos consumidores, que varia periodicamente e torna difícil encontrar uma relação estável entre o valor absoluto destas variáveis e a taxa de crescimento da economia. A diferença entre as variáveis SPAÍS e SFAM permite limitar este problema, dado que o "ponto de referência" utilizado para responder às duas questões tende a ser, em cada momento, muito semelhante.

Como se vê no gráfico seguinte (gráfico I), a diferença entre o valor das variáveis SPAÍS e SFAM atinge níveis não despiciendos, pelo que estamos perante um dado que é em si mesmo notório e que justifica um interesse adicional.

\footnotetext{
${ }^{3}$ De acordo com o Instituto Nacional de Estatística, o saldo de respostas extremas (sre) corresponde à diferença entre a percentagem de respostas de valoração positiva $\mathrm{e}$ de valoração negativa, ou seja, sre $=\%$ resp.(+) - \%resp.(-). No Inquérito Qualitativo de Conjuntura aos Consumidores existem questões com mais do que uma opção de natureza positiva/negativa. Nestes casos, às percentagens de resposta mais positivas/negativas é atribuído um peso de 1 e às restantes respostas um ponderador de 0,5 , ou seja, sre $=\left[\left(\%\right.\right.$ resp. $(++) * 1.0+\%$ resp. $\left.(+)^{*} 0.5\right)-(\%$ resp. $(-) * 0.5+\%$ resp. $(--$

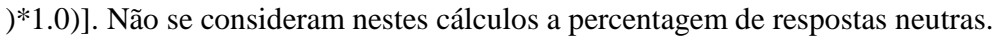




\section{Gráfico I}

Saldo das respostas extremas às questões sobre a situação económica do país (SPAÍS) e a situação financeira do agregado familiar (SFAM) nos próximos 12 meses e

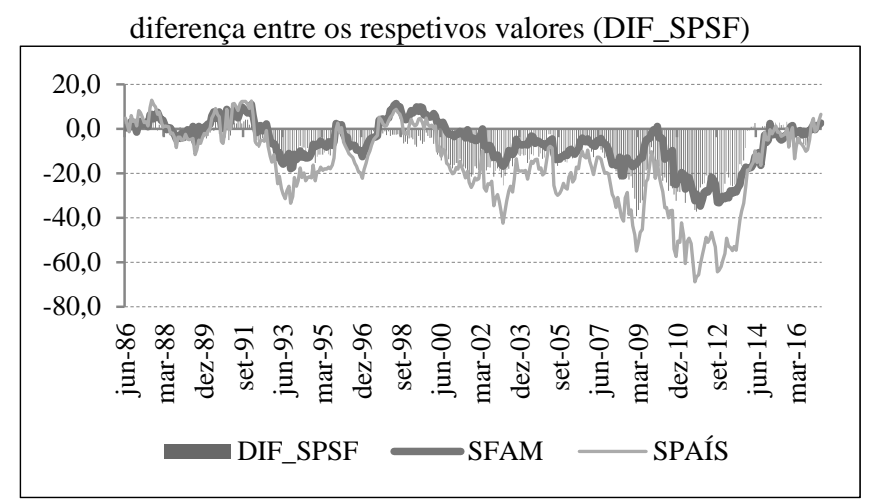

Fontes: Instituto Nacional de Estatística (Inquérito Qualitativo de Conjuntura aos Consumidores) e Directorate-General Financial and Economic Affairs (ECFIN)

Fica também evidente no gráfico que o valor absoluto das variáveis SPAÍS e SFAM é predominantemente negativo e que a diferença entre esses valores é substancialmente maior, em termos absolutos, quando estamos em ciclos negativos. Uma explicação simples para esta assimetria é a já mencionada "aversão às perdas". Neste estudo não exploramos este aspeto devido à necessidade de simplificar argumentos e limitar a dimensão do artigo ${ }^{4}$. No entanto, é muito possível que os resultados obtidos possam ser refinados com a inclusão nos modelos de uma distinção entre ciclos positivos e negativos.

\subsection{Crescimento Económico: Indicadores}

A medida existente teoricamente mais adequada para caracterizar o ciclo económico de forma instantânea é a taxa de crescimento do PIB trimestral real calculada em cadeia (isto é, face ao trimestre anterior). No entanto, esta taxa de crescimento apresenta flutuações significativas em Portugal (e noutros países, sobretudo com economias relativamente pequenas), dado que o valor do PIB num dado trimestre é facilmente influenciado por fenómenos pontuais e por efeitos de sazonalidade e de

\footnotetext{
${ }^{4}$ Diga-se, contudo, que existe já trabalho feito no âmbito do efeito assimétrico que más notícias e boas notícias exercem sobre o sentimento dos indivíduos (ver, por exemplo, Casey e Owen, 2013).
} 
calendário, cujo tratamento estatístico raramente consegue resolver de forma eficaz.

Como alternativa, é normal utilizar a taxa de crescimento do PIB trimestral real calculada em termos homólogos (isto é, face ao mesmo trimestre do ano anterior). Desta forma é possível suavizar algumas oscilações pontuais, sobretudo quando causadas por efeitos de calendário ou de sazonalidade. Apesar de tudo, subsistem problemas com este indicador, o primeiro dos quais é a sua vulnerabilidade a efeitos base e a anomalias pontuais nos trimestres de referência.

Por esta razão, alguns indicadores alternativos têm sido desenvolvidos no sentido de suavizar a medição do desempenho económico: são indicadores que utilizam como referência o comportamento real do PIB, mas adicionam métodos econométricos e outra informação para alisar os desvios pontuais face à tendência de curto e médio prazo do PIB. Um dos indicadores mais utilizados em Portugal para obter esta medida alisada do crescimento económico é o indicador coincidente do Banco de Portugal (IBP). O IBP tem a vantagem de ter uma periodicidade mensal (tal como os índices de confiança) e de ser uma estimativa relativamente fiável do crescimento instantâneo da economia, representando assim uma excelente caracterização do ciclo económico.

A sua principal desvantagem é a frequente revisão retrospetiva dos dados, sempre que os valores do PIB são corrigidos ou saem desalinhados com as expetativas. Por essa razão, neste estudo utilizamos apenas dados até 2016, evitando os riscos da muito provável revisão dos valores do crescimento económico relativos a 2017 (ainda bastante preliminares).

\section{CONFIANÇA DOS CONSUMIDORES E CRESCIMENTO ECONÓMICO}

\subsection{Resumo metodológico da análise}

Como se disse, o objetivo principal deste estudo é dar um contributo para a melhor utilização dos índices de confiança como indicadores do ciclo económico, valorizando em particular o papel do "efeito disponibilidade" ou, melhor, da nossa medida do "efeito disponibilidade": a diferença do saldo das respostas dadas às questões sobre a "situação económica no país nos próximos 12 meses" e a "situação financeira do agregado familiar nos próximos 12 meses".

Para isso, começamos por avaliar de que forma é que esta medida do "efeito disponibilidade" se correlaciona com o ciclo económico, em 
termos isolados e em comparação com os indicadores tradicionais de confiança.

Em função dos resultados realizamos uma série de repressões múltiplas, em que o crescimento económico é a variável endógena e as variáveis explicativas são os principais índices de confiança tradicionais e a nossa medida do "efeito disponibilidade".

Como se percebe, esta metodologia tem uma alcance parcial, quer pela sua simplicidade quer porque excluímos da análise outras variáveis relevantes para a identificação do ciclo económico. Apesar de tudo, comparando a robustez explicativa dos indicadores de confiança com e sem a inclusão do "efeito disponibilidade" podemos retirar algumas conclusões quanto à importância de adicionar este efeito ao estudo da relação entre os índices de confiança e a atividade económica, quer numa perspetiva coincidente quer avançada.

\subsection{Resultados com base no indicador coincidente do Banco de Portugal}

$\mathrm{O}$ primeiro conjunto de resultados que apresentamos neste artigo utiliza o indicador coincidente do Banco de Portugal (IBP) como medida do crescimento económico. As variáveis explicativas analisadas (em termos coincidentes e com desfasamento temporal) são o indicador de confiança dos consumidores (ICC), o saldo de respostas extremas à questão sobre a "situação económica no país nos próximos 12 meses" (SPAÍS), o saldo de respostas extremas à questão sobre a "situação financeira do agregado familiar nos próximos 12 meses" (SFAM) e a diferença entre estes dois últimos valores, calculada como SPAÍS menos SFAM (DIF_SPSF). A nossa hipótese de trabalho é que quanto mais positivo (ou menos negativo) for o valor desta diferença, maior o crescimento económico associado. Além disso, esperamos que esta relação seja significativa em termos coincidentes, mas também em termos avançados, dada a influência que as expetativas dos consumidores podem ter no seu comportamento e, por isso, na atividade económica.

Os dados utilizados são mensais e cobrem o período 1986-2016. Contudo, iremos testar também os resultados para o horizonte temporal 1986-2007, excluindo assim da amostra o ciclo altamente recessivo de 2008-2009 e o período de vigência do Programa de Ajustamento Económico e Financeiro, a que Portugal esteve sujeito (2011-2013), dado que os valores observados nestes anos são claros outliers no âmbito das séries utilizadas, podendo influenciar decisivamente os resultados. 
Para o período 1986-2016 (amostra completa) a primeira conclusão é de que os indicadores de confiança analisados apresentam uma correlação contemporânea bastante elevada com o IBP, sendo que os indicadores tradicionais (i.e., utilizados pelo INE) apresentam valores de correlação ligeiramente superiores ao apresentado por DIF_SPSF (ver Tabela I). Ou seja, não há evidência suficiente para dizer que DIF_SPSF é, por si só, um melhor indicador do ciclo económico do que as variáveis utilizadas para construir o índice de confiança dos consumidores. Este resultado não põe em causa o nosso argumento, dado que a correlação contemporânea entre DIF_SPSF e IBP é também elevada $(0,76)$, justificando que se explore em maior detalhe a relevância de DIF_SPSF. Além disso, um dado particularmente interessante é que a correlação entre DIF_SPSF e IBP não diminui com o desfasamento temporal, ao contrário do que se passa com as outras variáveis, o que reforça o potencial de DIF_SPSF como indicador avançado. Note-se que o coeficiente máximo da correlação entre DIF_SPSF e IBP verifica-se com um desfasamento de dois e três meses, com um valor de 0,77 .

O quadro seguinte (Tabela I) ilustra os principais valores da correlação contemporânea e desfasada entre o indicador coincidente do Banco de Portugal e os indicadores de confiança utilizados neste estudo.

\section{Tabela I}

Correlação entre o indicador coincidente do Banco de Portugal e variáveis de confiança dos consumidores (amostra 1986-2016)

\begin{tabular}{lcccc}
\hline & ICC & SFAM & SPAÍS & DIF_SPSF \\
\hline $\begin{array}{l}\text { Correlação } \\
\text { Contemporânea }\end{array}$ & 0,85 & 0,82 & 0,85 & 0,76 \\
\hline $\begin{array}{l}\text { Máxima } \\
\text { Correlação }\end{array}$ & 0,85 & 0,82 & 0,85 & 0,77 \\
& $\left(\mathrm{ICC}_{\mathrm{t}}\right)$ & $\left(\mathrm{SF}_{\mathrm{t}}\right)$ & $\left(\mathrm{SP}_{\mathrm{t} / \mathrm{t}-1)}\right.$ & $\left(\mathrm{DIF}_{-} \mathrm{SPSF}_{\mathrm{t}-2 / \mathrm{t}-}\right.$ \\
& & & & $3)$ \\
\hline
\end{tabular}

Nota: t identifica o mês da observação

A segunda conclusão é que a incorporação na análise de DIF_SPSF aumenta o poder explicativo das variáveis de confiança relativamente ao IBP, quer em termos coincidentes quer avançados. O quadro seguinte (Tabela II) sintetiza esta conclusão, com base em regressões lineares que têm como variável dependente o IBP e como variáveis independentes os indicadores de confiança já mencionados e DIF_SPSF. 


\section{Tabela II}

Relação entre o indicador coincidente do Banco de Portugal e variáveis de confiança dos consumidores (amostra 1986-2016)

\begin{tabular}{|c|c|c|c|c|c|c|}
\hline Variáveis & (1) & (2) & (3) & (4) & (5) & (6) \\
\hline Constante & $\begin{array}{c}4,778 * * \\
(38,9)\end{array}$ & $\begin{array}{r}4,248 * * \\
(19,97) \\
\end{array}$ & $\begin{array}{c}4,744 * * \\
(40,3)\end{array}$ & $\begin{array}{c}4,565 * * \\
(25,58) \\
\end{array}$ & $\begin{array}{l}4,768 * * \\
(36,15) \\
\end{array}$ & $\begin{array}{l}4,59 * * \\
(34,94) \\
\end{array}$ \\
\hline $\mathrm{ICC}_{\mathrm{t}}$ & $\begin{array}{c}0,125^{* *} \\
(14,63)\end{array}$ & $\begin{array}{c}0,079 * * \\
(4,52)\end{array}$ & $\begin{array}{c}0,114 * * \\
(14,97)\end{array}$ & $\begin{array}{c}0,096^{* * *} \\
(6,35)\end{array}$ & & \\
\hline SFAM $_{\mathrm{t}}$ & & $\begin{array}{c}0,059 * * \\
(3,03)\end{array}$ & & & & \\
\hline SPAIÍS $_{\mathrm{t}}$ & & & & $\begin{array}{l}0,018 \\
(1,33)\end{array}$ & & \\
\hline DIF_SPSF $_{t}$ & $\begin{array}{c}0,039 * * \\
(3,33)\end{array}$ & $\begin{array}{c}0,046^{* * *} \\
(3,84)\end{array}$ & & & & \\
\hline $\operatorname{ICC}_{(\mathrm{t}-3)}$ & & & & & $\begin{array}{l}0,143 * * \\
(27,19) \\
\end{array}$ & $\begin{array}{l}0,102 * * \\
(11,31) \\
\end{array}$ \\
\hline DIF_SPSF $_{(t-3)}$ & & & $\begin{array}{c}0,059 * * \\
(5,60)\end{array}$ & $\begin{array}{c}0,049 * * \\
(3,78)\end{array}$ & & $\begin{array}{c}0,067 * * \\
(5,37)\end{array}$ \\
\hline $\mathrm{R}^{2}$ & 0,73 & 0,74 & 0,75 & 0,75 & 0,67 & 0,70 \\
\hline $\begin{array}{l}\text { Número de } \\
\text { observações }\end{array}$ & 367 & 367 & 364 & 364 & 364 & 364 \\
\hline
\end{tabular}

Notas: a variável dependente é o indicador coincidente do Banco de Portugal; o subíndice t identifica o mês da observação. t-estatísticas entre parênteses.

*significativo a $5 \% * *$ significativo a $1 \%$

As regressões (1) e (2) ilustram o contributo de DIF_SPSF para o reforço da relação contemporânea entre os indicadores de confiança tradicionais e o crescimento económico. As regressões (3) a (6) mostram o contributo de DIF_SPSF, como indicador avançado (desfasamento de 3 meses), para o reforço da correlação, coincidente e desfasada, entre os valores da confiança e o ritmo de crescimento económico.

Dito isto, um ponto a realçar é que os resultados baseados nesta amostra não permitem estabelecer, ainda, de forma conclusiva a vantagem de utilizar DIF_SPSF em vez de apenas SPAÍS. Estas duas variáveis apresentam, nesta amostra, uma elevada correlação contemporânea, o que significa que, nalguns testes, podemos substituir DIF_SPSF por SPAÍS sem alterar substancialmente os resultados. Contudo, há argumentos que nos permitem continuar a supor que DIF_SPSF é, além de uma métrica mais intuitiva do "efeito disponibilidade", um indicador mais robusto da relação entre a confiança e o crescimento económico do que SPAÍS. Desde logo porque DIF_SPSF é menos influenciado pelo "efeito referência", mas também porque a elevada correlação entre DIF_SPSF e SPAÍS observada nesta amostra, 
não é replicada na mesma dimensão noutras amostras que excluem os períodos de extrema crise já mencionados (2008-2009 e 2011-2013).

A nossa convicção parece ser validada pelos resultados que apresentamos a seguir com base na amostra limitada (1986-2007) e, mais à frente, com base na utilização da taxa de crescimento homólogo do PIB como indicador do ciclo económico. No primeiro caso, porque expurgamos da análise o impacto das observações extremas registadas entre 2008 e 2013, que afetaram bastante a variável SPAÍS e, consequentemente, DIF_SPSF (aumentando anormalmente a correlação entre ambas). No segundo caso, cremos que devido à própria construção do indicador coincidente do Banco de Portugal, que recorre aos índices de confiança para alisar os dados de crescimento do produto interno bruto.

\section{Amostra Limitada (1986-2007)}

Pelas razões já mencionadas, repetimos a análise anterior para uma amostra mais reduzida no tempo: entre 1986 e 2007 (amostra limitada). Os resultados com esta amostra reforçam a importância da variável DIF_SPSF, sugerindo que a relevância estatística dos outros indicadores de confiança assenta, em parte, nos dados extremos observados entre 2008 e 2013. Nos quadros seguintes (Tabela III e Tabela IV) sintetizamos os principais resultados para esta amostra limitada, que evidenciam o papel acrescido de DIF_SPSF na identificação do ciclo económico.

\section{Tabela III}

Correlação entre o indicador coincidente do Banco de Portugal e variáveis de confiança dos consumidores (amostra 1986-2007)

\begin{tabular}{lcccc}
\hline & ICC & SFAM & SPAÍ́S & DIF_SPSF \\
\hline $\begin{array}{l}\text { Correlação } \\
\begin{array}{l}\text { Contemporânea } \\
\text { Correlação com }\end{array}\end{array}$ & 0,80 & 0,76 & 0,80 & 0,71 \\
$\begin{array}{l}\text { desfasamento de } \\
3 \text { meses }\end{array}$ & 0,70 & 0,66 & 0,73 & 0,69 \\
\hline
\end{tabular}


Tabela IV

Relação entre o indicador coincidente do Banco de Portugal e variáveis de confiança dos consumidores (amostra 1986-2007)

\begin{tabular}{|c|c|c|c|c|c|c|}
\hline & (1) & (2) & (3) & (4) & (5) & (6) \\
\hline Constante & $\begin{array}{l}4,915^{* *} \\
(41,45) \\
\end{array}$ & $\begin{array}{l}5,005^{* *} \\
(20,16) \\
\end{array}$ & $\begin{array}{l}4,941 * * \\
(42,09) \\
\end{array}$ & $\begin{array}{l}4,813 \text { *** } \\
(28,38) \\
\end{array}$ & $\begin{array}{l}4,633 * * \\
(31,09) \\
\end{array}$ & $\begin{array}{l}4,618^{* * *} \\
(33,53) \\
\end{array}$ \\
\hline $\mathrm{ICC}_{\mathrm{t}}$ & $\begin{array}{l}0,099 * * \\
(12,04)\end{array}$ & $\begin{array}{c}0,106 * * \\
(5,67)\end{array}$ & $\begin{array}{l}0,101 * * \\
(13,27)\end{array}$ & $\begin{array}{c}0,089 * * \\
(6,28)\end{array}$ & & \\
\hline SPAÍ́S $_{\mathrm{t}}$ & & $\begin{array}{l}-0,012 \\
(-0,41)\end{array}$ & & $\begin{array}{l}0,016 \\
(1,04)\end{array}$ & & \\
\hline DIF_SPSF $_{t}$ & $\begin{array}{c}0,077 * * \\
(5,77) \\
\end{array}$ & $\begin{array}{c}0,089 * * \\
(2,76) \\
\end{array}$ & & & & \\
\hline $\mathrm{ICC}_{(\mathrm{t}-3)}$ & & & & & $\begin{array}{c}0,118^{* * *} \\
(15,82) \\
\end{array}$ & $\begin{array}{c}0,103^{* *} \\
(6,67) \\
\end{array}$ \\
\hline DIF_SPSF(t-3) $_{1}$ & & & $\begin{array}{c}0,078^{* * *} \\
(6,40)\end{array}$ & $\begin{array}{c}0,068^{* * *} \\
(4,21)\end{array}$ & & $\begin{array}{c}0,073^{* *} \\
(5,56) \\
\end{array}$ \\
\hline $\mathrm{R}^{2}$ & 0,68 & 0,68 & 0,69 & 0,69 & 0,50 & 0,57 \\
\hline $\begin{array}{l}\text { Número de } \\
\text { observações }\end{array}$ & 259 & 259 & 256 & 256 & 256 & 256 \\
\hline
\end{tabular}

Notas: a variável dependente é o indicador coincidente do Banco de Portugal; o subíndice $\mathrm{t}$ identifica o mês da observação. t-estatísticas entre parênteses.

*significativo a $5 \% * *$ significativo a $1 \%$

O primeiro aspeto a salientar nestes resultados é a menor correlação entre os indicadores de confiança e o IBP, bem como o menor coeficiente de determinação ( $r$ quadrado) das regressões. Este era um resultado esperado, uma vez que as quebras de atividade económica e de confiança verificadas no período 2008-2013 foram de tal forma singulares (em termos históricos e na sua dimensão) que, do ponto de vista estatístico, acabaram por reforçar a correlação entre estas variáveis. Este efeito verificou-se, aliás, com outras variáveis (cuja relação entre si é questionável) que foram severamente e invulgarmente afetadas pela crise financeira e orçamental (por exemplo, as exportações, a taxa de desemprego, o volume de concessão de crédito e as taxas de juro da dívida pública). Apenas para ter uma noção do impacto estatístico desta crise, devido aos valores anormais verificados entre 2008 e 2013 o próprio tratamento da sazonalidade continua a ser um problema reconhecido nas contas nacionais de diversos países.

Neste contexto, é ainda mais importante constatar que DIF_SPSF mantém um contributo importante no sentido de reforçar a relação entre os indicadores tradicionais de confiança e o IBP, quer em termos coincidentes quer avançados. Acresce que, além da menor correlação contemporânea entre DIF_SPSF e SPAÍS registada nesta amostra, fica 
também evidente o maior poder explicativo de DIF_SPSF face a SPAÍS em todas as regressões efetuadas, algo que não sucede no caso da amostra completa.

Um dado particular a retirar dos resultados obtidos com a amostra limitada é que a utilização isolada dos índices tradicionais de confiança como indicadores avançados perde significado estatístico: os coeficientes de correlação máximos entre o IBP e as variáveis ICC, SPAÍS e SFAM são de natureza coincidente e diminuem muito com o tempo. Neste sentido, é de realçar o impacto positivo de adicionar à análise a variável DIF_SPSF como indicador avançado: vide regressões (5) e (6).

\subsection{Resultados com base na taxa de crescimento do PIB}

$\mathrm{O}$ gráfico seguinte (gráfico II) relaciona o crescimento do PIB trimestral real em termos homólogos com a correspondente média trimestral do indicador coincidente do Banco de Portugal. Como se pode constatar, o comportamento da taxa de crescimento do PIB é mais irregular do que a trajetória do indicador coincidente do Banco de Portugal. Apesar de tudo, é de todo o interesse tentar validar os resultados obtidos até agora, utilizando como referência os dados oficiais de crescimento económico 5 .

\footnotetext{
${ }^{5}$ As taxas de crescimento do PIB trimestral até 1995 são calculadas pelo Banco de Portugal e publicadas anualmente, conjuntamente com o Boletim Económico de Junho, num anexo intitulado "séries trimestrais para a economia portuguesa". Os dados posteriores a 1995 são calculados pelo Instituto Nacional de Estatística e divulgados nas suas Contas Nacionais Trimestrais. O Banco de Portugal faz a reconciliação destas duas séries, respeitando os valores pós-1995 apresentados pelo INE.
} 


\section{Gráfico II}

A taxa de crescimento homólogo do PIB trimestral real e o indicador coincidente do Banco de Portugal (média trimestral)

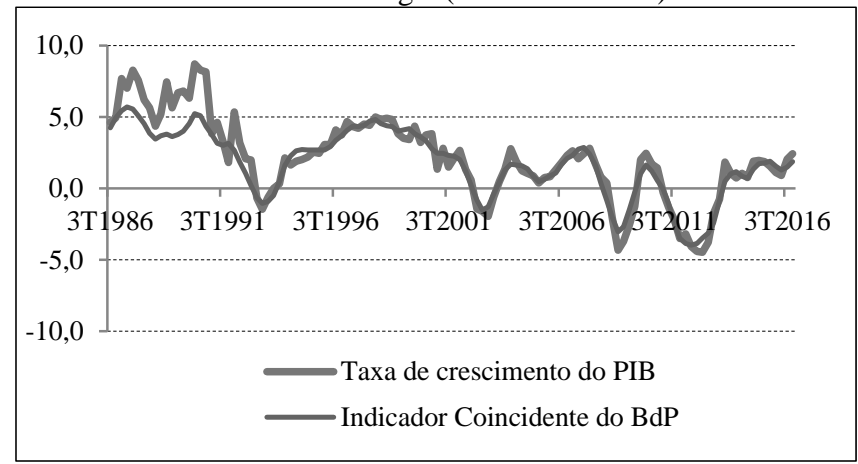

Fontes: Banco de Portugal e Instituto Nacional de Estatística

Apesar da natureza mais irregular da taxa de crescimento do PIB e do desfasamento existente até 1990 entre esta variável e o IBP, os resultados obtidos com a utilização da taxa de crescimento do PIB trimestral como variável dependente são muito semelhantes - em termos quantitativos e qualitativos - aos resultados obtidos com a utilização do indicador coincidente do Banco de Portugal. Este facto é ilustrado nos quadros seguintes (Tabelas V e VI), para o caso da amostra completa (19862016).

\section{Tabela V}

Correlação entre o crescimento homólogo do PIB trimestral e variáveis de confiança dos consumidores (amostra 1986-2016)

\begin{tabular}{lcccc}
\hline & ICC & SFAM & SPAÍS & DIF_SPSF \\
\hline $\begin{array}{l}\text { Correlação } \\
\text { Contemporânea }\end{array}$ & 0,82 & 0,77 & 0,81 & 0,75 \\
\hline Máxima & 0,82 & 0,77 & 0,81 & 0,76 \\
Correlação & $\left(\mathrm{ICC}_{\mathrm{t}}\right)$ & $\left(\mathrm{SF}_{\mathrm{t}}\right)$ & $\left(\mathrm{SP}_{\mathrm{t}}\right)$ & $\left(\mathrm{DIF} \mathrm{SPSF}_{\mathrm{t}-1}\right)$ \\
\hline
\end{tabular}

Nota: o subíndice $t$ identifica o trimestre da observação 


\section{Tabela VI}

Relação entre o crescimento homólogo do PIB trimestral e variáveis de confiança dos consumidores (amostra 1986-2016)

\begin{tabular}{|c|c|c|c|c|c|c|}
\hline Variáveis & (1) & (2) & (3) & (4) & (5) & (6) \\
\hline Constante & $\begin{array}{l}5,789 * * \\
(19,48)\end{array}$ & $\begin{array}{l}5,700 * * \\
(10,65)\end{array}$ & $\begin{array}{l}5,700 * * \\
(10,65)\end{array}$ & $\begin{array}{l}4,813 * * \\
(28,38) \\
\end{array}$ & $\begin{array}{l}5,851 * * \\
(18,89)\end{array}$ & $\begin{array}{c}5,588 * * \\
(18,2)\end{array}$ \\
\hline $\mathrm{ICC}_{\mathrm{t}}$ & $\begin{array}{c}0,142^{* * *} \\
(6,72)\end{array}$ & $\begin{array}{c}0,106^{* * *} \\
(5,67)\end{array}$ & $\begin{array}{c}0,106^{* * *} \\
(5,67)\end{array}$ & $\begin{array}{c}0,089 * * \\
(6,28)\end{array}$ & & \\
\hline $\mathrm{SFAM}_{\mathrm{t}}$ & & $\begin{array}{l}0,01 \\
(0,2)\end{array}$ & & & & \\
\hline SPAÍ́S $_{\mathrm{t}}$ & & & $\begin{array}{l}0,01 \\
(0,2)\end{array}$ & $\begin{array}{c}-0,01 \\
(-0,28)\end{array}$ & & \\
\hline DIF_SPSF $_{t}$ & $\begin{array}{l}0,064^{*} \\
(2,16)\end{array}$ & $\begin{array}{l}0,065^{*} \\
(2,16)\end{array}$ & $\begin{array}{l}0,055^{*} \\
(1,03)\end{array}$ & & & \\
\hline $\operatorname{ICC}_{(\mathrm{t}-1)}$ & & & & & $\begin{array}{l}0,176^{* * *} \\
(14,21)\end{array}$ & $\begin{array}{c}0,114 * * \\
(5,26)\end{array}$ \\
\hline DIF_SPSF $_{(\mathrm{t}-1)}$ & & & & $\begin{array}{c}0,096^{*} \\
(2,56) \\
\end{array}$ & & $\begin{array}{c}0,103 * * \\
(3,38) \\
\end{array}$ \\
\hline $\mathrm{R}^{2}$ & 0,68 & 0,68 & 0,68 & 0,69 & 0,63 & 0,66 \\
\hline $\begin{array}{l}\text { Número de } \\
\text { observações }\end{array}$ & 122 & 122 & 122 & 121 & 121 & 121 \\
\hline
\end{tabular}

Notas: a variável dependente é o indicador coincidente do Banco de Portugal; o subíndice $t$ identifica o trimestre da observação. t-estatísticas entre parênteses.

*significativo a $5 \% * *$ significativo a $1 \%$

Como esperado, é visível uma diminuição dos coeficientes de correlação entre o crescimento do PIB e as variáveis de confiança tradicionais, face aos testes em que IBP foi usado como indicador do crescimento. Note-se, contudo, que a correlação (contemporânea e avançada) entre o crescimento do PIB e a variável DIF_SPSF é praticamente igual à verificada entre IBP e DIF_SPSF, o que ajuda a sustentar a robustez da variável DIF_SPSF no contexto desta análise.

No restante, os resultados são muito semelhantes, talvez com exceção da ligeira quebra de significado estatístico da variável DIF_SPSF como indicador coincidente do ciclo (mas sem pôr em causa o maior poder explicativo desta variável face a SPAÍS), sendo que a relevância de DIF_SPSF como indicador avançado mantém-se forte. Em suma, os resultados estão em linha com os nossos argumentos.

\section{Amostra Limitada (1986-2007)}

Os resultados obtidos com dados entre 1986-2007 são apresentados nos quadros seguintes (Tabela VII e Tabela VIII). 
Tabela VII

Correlação entre o crescimento homólogo do PIB trimestral e variáveis de confiança dos consumidores (amostra 1986-2007)

\begin{tabular}{llccc}
\hline & ICC & SFAM & SPAÍS & DIF_SPSF \\
\hline $\begin{array}{l}\text { Correlação } \\
\text { Contemporânea }\end{array}$ & 0,77 & 0,71 & 0,79 & 0,74 \\
\hline $\begin{array}{l}\text { Correlação com } \\
\text { desfasamento de }\end{array}$ & 0,70 & 0,64 & 0,75 & 0,74 \\
1 trimestre & & & & \\
\hline
\end{tabular}

\section{Tabela VIII}

Relação entre o crescimento homólogo do PIB trimestral e variáveis de confiança dos consumidores (amostra 1986-2007)

\begin{tabular}{|c|c|c|c|c|c|c|}
\hline & $(1)$ & $(2)$ & (3) & $(4)$ & $(5)$ & $(6)$ \\
\hline Constante & $\begin{array}{l}6,28 * * \\
(21,14) \\
\end{array}$ & $\begin{array}{l}7,348^{* * *} \\
(11,17) \\
\end{array}$ & $\begin{array}{l}7,499 * * \\
(11,52) \\
\end{array}$ & $\begin{array}{l}5,965 * * \\
(16,19) \\
\end{array}$ & $\begin{array}{r}5,967 * * \\
(18,42) \\
\end{array}$ & $\begin{array}{l}7,302 * * \\
(10,25) \\
\end{array}$ \\
\hline $\mathrm{ICC}_{\mathrm{t}}$ & $\begin{array}{c}0,117 * * \\
(5,57)\end{array}$ & $\begin{array}{c}0,199 * * \\
(4,00)\end{array}$ & $\begin{array}{c}0,209 * * \\
(4,29) \\
\end{array}$ & & & \\
\hline SFAM $_{\mathrm{t}}$ & & $\begin{array}{l}-0,138 \\
(-1,81) \\
\end{array}$ & $\begin{array}{c}-0,157^{*} \\
(-2,07) \\
\end{array}$ & & & \\
\hline DIF_SPSF $_{t}$ & $\begin{array}{c}0,148^{* * *} \\
(4,29)\end{array}$ & $\begin{array}{c}0,156^{* * *} \\
(4,54)\end{array}$ & & & & \\
\hline $\operatorname{ICC}_{(t-1)}$ & & & & $\begin{array}{c}0,165 * * \\
(8,93) \\
\end{array}$ & $\begin{array}{c}0,083 * * \\
(3,62)\end{array}$ & $\begin{array}{c}0,185 * * \\
(3,45) \\
\end{array}$ \\
\hline SPAÍS $_{(t-1)}$ & & & & & & $\begin{array}{c}-0,173^{*} \\
(-2,09)\end{array}$ \\
\hline DIF_SPSF $_{(\mathrm{t}-1)}$ & & & $\begin{array}{c}0,164 * * \\
(4,96)\end{array}$ & & $\begin{array}{l}0,19 * * \\
(5,03)\end{array}$ & $\begin{array}{c}0,372 * * \\
(3,93)\end{array}$ \\
\hline $\mathrm{R}^{2}$ & 0,67 & 0,68 & 0,70 & 0,49 & 0,61 & 0,63 \\
\hline $\begin{array}{l}\text { Número de } \\
\text { observações }\end{array}$ & 86 & 86 & 85 & 85 & 85 & 85 \\
\hline
\end{tabular}

Notas: a variável dependente é o indicador coincidente do Banco de Portugal; o subíndice $\mathrm{t}$ identifica o trimestre da observação. t-estatísticas entre parênteses.

*significativo a $5 \% * *$ significativo a $1 \%$

Como esperado, a utilização da amostra limitada reforça transversalmente a relevância de DIF_SPSF, como indicador coincidente e avançado do ciclo económico. Particularmente significativo é o facto de DIF_SPSF passar a ter um coeficiente de correlação contemporâneo com a taxa de crescimento do PIB mais elevado do que SFAM e um coeficiente de correlação desfasado com o crescimento do PIB superior ou idêntico ao dos indicadores de confiança tradicionais (ver Tabela VII). Este último facto traduz-se, depois, num contributo superior de DIF_SPSF para a relevância de utilizar os dados da confiança como 
indicadores avançados do ciclo: vide regressões (4), (5) e (6) na Tabela VIII.

\section{CONCLUSÃO}

A motivação inicial deste artigo é apresentar e explorar uma característica típica dos inquéritos de confiança aos consumidores: a diferença entre as respostas dadas à questão sobre "a situação financeira do lar" e sobre a "situação económica do país". Há, naturalmente, várias explicações intuitivas e lógicas para esta diferença. Neste artigo optamos por valorizar o "efeito disponibilidade", que no essencial atribui esta diferença ao facto de os indivíduos basearem as suas respostas à questão sobre a "situação económica do país" não apenas na sua própria experiência, mas também na informação que recolhem do exterior, quer diretamente quer através da imprensa ou do discurso político. É um argumento simples e que, na prática, agrega muitas das explicações possíveis para esta diferença entre as respostas.

Em termos gerais, o "efeito disponibilidade" provoca muitas vezes enviesamentos nas perceções dos indivíduos relativamente a determinados eventos, levando por exemplo à sobre estimação da probabilidade de eventos raros e à subestimação da probabilidade de eventos frequentes, dado que a imprensa tende a dar maior saliência aos fenómenos de maior impacto noticioso, que são muitas vezes os eventos mais estranhos ou mais raros.

Contudo, no caso específico do ciclo económico é de esperar que haja, de facto, uma correlação forte entre a dimensão do "efeito disponibilidade" e a situação económica, seja por via coincidente ou avançada. A razão para esta hipótese é que o tipo e a frequência de notícias dominantes sobre a situação económica acompanham, frequentemente, a natureza do ciclo. $\mathrm{E}$ mesmo que isto não seja totalmente verdade, a influência que a perceção dos agentes tem no seu próprio comportamento tende a funcionar como uma "profecia autorealizável", no sentido em que se estiver enraizada a ideia de que há um importante abrandamento económico, o comportamento dos agentes tende a ser propício à ocorrência efetiva desse abrandamento (e o mesmo para o caso de uma aceleração da economia) ${ }^{6}$.

\footnotetext{
${ }^{6}$ Este argumento não é novo. Em particular, é conhecida a prática de contar o número de vezes que aparece a palavra "recessão" na imprensa como forma de estimar a
} 
Perante isto, o nosso argumento principal é que a dimensão do "efeito disponibilidade" e a natureza do ciclo económico estão altamente correlacionados, tanto mais que a métrica que utilizamos para medir o "efeito disponibilidade" evita o "efeito referência", ao contrário do que sucede com o índice de confiança dos consumidores e os componentes deste índice. Esta correlação é válida em termos contemporâneos, mas também em termos avançados, devido ao impacto que o "efeito disponibilidade" tem no comportamento dos indivíduos. Os resultados sugerem a validade desta hipótese, com especial ênfase na natureza avançada do indicador que utilizamos para medir o "efeito disponibilidade".

Uma motivação adicional para este estudo prende-se com a vontade de expor o leitor ao papel que determinados fenómenos comportamentais podem ter na análise económica, nomeadamente no âmbito da macroeconomia e do crescimento económico. Este artigo é sobretudo simbólico a esse respeito, dado que os resultados obtidos e os métodos utilizados não são suficientes para retirar lições de política económica, nem para propor um novo modelo de indicador avançado do ciclo. Apesar de tudo, julgamos que as conclusões apresentadas sugerem que algum trabalho adicional pode ser feito nesse sentido e que este é, pelo menos, um artigo sensibilizador da importância de aprofundar os estudos sobre a relação entre os fundamentos da economia comportamental e a macroeconomia.

\section{FONTES E BIBLIOGRAFIA}

Aarle, B., Moons, C. (2017). Sentiment and Uncertainty Fluctuations and Their Effects on the Euro Area Business Cycle, Journal of Business Cycle Research, 13(2), 225-251.

Acemoglu, D., Scott, A. (1994). Consumer Confidence and Rational Expectations: Are Agents' Beliefs Consistent with the Theory?, Economic Journal, 104:422, 1-19.

Assarsson, B., Osterholm, P. (2015). Do Swedish Consumer Confidence

Indicators Do What They Are Intended to Do?. Applied Economics Quarterly, 61(4), 391-404.

probabilidade de estarmos perante um ciclo económico negativo, no presente ou no futuro próximo. 
Banco de Portugal (2018). Indicadores Coincidentes. https://www.bportugal.pt/publications/banco-de-portugal/all/116.

Acedido em 22 de março de 2018.

Banco de Portugal (2018). Boletim Económico.

https://www.bportugal.pt/publications/banco-de-portugal/all/381.

Acedido em 22 de março de 2018.

Bateman, L., Munro, A., Rhodes, B., Starmer, C., Sudgen, R. (1997). A Test of the Theory of Reference-Dependent Preferences, Quarterly Journal of Economics, 112(2), 479-505.

Bondt, J., Schiaffi, S. (2015). Confidence Matters for Current Economic Growth: Empirical Evidence for the Euro Area and the United States, Social Science Quarterly, 96(4), 1027-1040.

Bowman, D., Minehart, D., Rabin, M. (1999). Loss Aversion in a Consumption-Savings Model, Journal of Economic Behavior and Organization, 38(2), 155-78.

Carroll, C., Fuhrer, J., Wilcox, D. (1994). Does Consumer Sentiment Forecast Household Spending? If So, Why? American Economic Review, 84(5), 1397-1408.

Casey, G., Owen, A. (2013). Good News, Bad News, and Consumer Confidence, Social Science Quarterly, 94(1), 292-315.

Dees, S., Brinca, P. S. (2013). Consumer Confidence as a Predictor of Consumption Spending: Evidence for the United States and the Euro Area, International Economics, 134, 1-14.

Directorate General for Economic and Financial Affairs (2018). Business and Consumer Surveys. https://ec.europa.eu/info/business-economyeuro/indicators-statistics/economic-databases/business-andconsumer-surveys_en. Acedido em 22 de março de 2018.

Dominitz, J., Manski, C. (2004). How Should We Measure Consumer Confidence?, Journal of Economic Perspectives, 18(2), 51-66.

Gilovich, T., Griffin, D. W., Kahneman, D. (eds) (2002). Heuristics and Biases: The Psychology of Intuitive Judgment. Great Britain: Cambridge University Press.

Golinelli, R., Parigi, G. (2004). Consumer Sentiment and Economic Activity: A Cross Country Comparison, Journal of Business Cycle Measurement and Analysis, 2004(2), 147-170.

Greifeneder, R., Bless, H. (2007). Relying on Accessible Content Versus Accessibility Experiences: The Case of Processing Capacity, Social Cognition, 25, 853-881. 
Instituto Nacional de Estatística (2018). Contas Nacionais. https://www.ine.pt/xportal/xmain?xpid=INE\&xpgid=ine_cnacionais. Acedido em 22 de março de 2018.

Instituto Nacional de Estatística (2018). Inquéritos de Conjuntura às Empresas e aos Consumidores.

https://www.ine.pt/xportal/xmain?xpid=INE\&xpgid=ine_destaques\& DESTAQUESdest_boui $=308000936 \& D E S T A Q U E S m o d o=2$.

Acedido em 22 de março de 2018.

Kahneman, D. (2011). Thinking, fast and slow. Great Britain: Allen Lane. Kahneman, D., Knetsch, J. L., Thaler, R. (1991). Anomalies: The Endowment Effect, Loss Aversion, and Status Quo Bias, Journal of Economic Perspectives, 5(1), 193-206.

Kahneman, D., Slovic, P., Tversky, A. (eds) (1982). Judgment Under Uncertainty: Heuristics and Biases. Great Britain: Cambridge University Press.

Kahneman, D., Tversky, A. (1979). Prospect Theory: An Analysis of Decision Under Risk, Econometrica, 47(2), 263-291.

Kahneman, D., Tversky, A. (1984). Choices, Values and Frames, American Psychologist, 39(4), 341-350.

Lewis, M. (2017). The Undoing Project: A Friendship that Changed the World. Great Britain: Allen Lane.

Ludvigson, S. C. (2004). Consumer Confidence and Consumer Spending, Journal of Economic Perspectives, 18(2), 29-50.

Matsusaka, J., Sbordone, A. (1995). Consumer Confidence and Economic Fluctuations, Economic Inquiry, 33(2), 296-318.

Organization for Economic Co-operation and Development, OCDE (2018). Leading indicators and tendency surveys. http://www.oecd.org/sdd/leading-indicators/. Acedido em 22 de março de 2018.

Rabin, M. (1998). Psychology and Economics, Journal of Economic Literature, 36(1), 11-46.

Rua, A. (2004). Um novo indicador coincidente para a economia portuguesa. Boletim Económico do Banco de Portugal, junho, 21-29.

Schwarz, N., Bless, H., Strack, F., Klumpp, G., Rittenauer-Schatka, H., Simons, A. (1991). Ease of Retrieval as Information: Another Look at the Availability Heuristic. Journal of Personality and Social Psychology, 61(2), 195-202.

Tversky, A., Kahneman, D. (1973). Availability: A Heuristic for Judging Frequency and Probability, Cognitive Psychology, 5, 207-232. 
Tversky, A., Kahneman, D. (1974). Judgment Under Uncertainty: Heuristics and Biases, Science, 185, 1124-1131.

Tversky, A., Kahneman, D. (1981). The Framing of Decisions and the Psychology of Choice, Science, 211, 453-458.

Tversky, A., Kahneman, D. (1991). Loss Aversion in Riskless Choice: A Reference-Dependent Model, Quarterly Journal of Economics, 106(4), 1039-1061.

Wanke, M. Schwarz, N., Bless, H. (1995). The Availability Heuristic Revisited: Experienced Ease of Retrieval in Mundane Frequency Estimates, Acta Psychologica, 89, 83-90. 